\title{
Validación de una escala de perfeccionismo en niños peruanos
}

\author{
Validation of a Scale of Perfectionism in Peruvian Children
}

\author{
José Ventura-León ${ }^{1}$ \\ Stefani Jara-Avalos ${ }^{2}$ \\ Carmen Garcia-Pajuelo ${ }^{3}$ \\ Carlos Ortiz-Saenz ${ }^{4}$
}

Universidad Privada del Norte, Perú

\begin{abstract}
Resumen. Este artículo validó la Escala de Perfeccionismo Infantil (EPI) de Oros en una población infantil peruana. Se contó con la participación de 1084 niños con edades comprendidas entre los 7 y 12 años $(M=9.75$; $D E=1.23)$ de siete instituciones educativas, privadas y públicas. El Análisis Factorial Exploratorio reveló una escala bidimensional oblicua (Reacción ante el fracaso y Autodemandas); no obstante, tres ítems tuvieron una carga < .30. El Análisis Factorial Confirmatorio corroboró la estructura bidimensional oblicua con la salvedad de errores correlacionados. La fiabilidad fue estimada mediante el coeficiente Omega $(\omega=.810)$. Se concluye que la EPI cuenta con evidencia de validez y fiabilidad de las puntuaciones.
\end{abstract}

Palabras clave. Perfeccionismo, niños, validación, fiabilidad.

Abstract. This article discusses the validation of the Oros' Child Perfectionism Scale (EPI) in a Peruvian child population. A total of 1084 children aged 7 to 12 years $(M=9.75, S D=1.23)$ from seven private and public educational institutions participated. Exploratory Factor Analysis revealed an oblique two-dimensional scale (Reaction to failure and Self-orders); however, three items had a load <. 30. The Confirmatory Factor Analysis corroborated the bidimensional oblique structure with the exception of correlated errors. Reliability was estimated using the Omega coefficient $(\omega=.810)$. It is concluded that the EPI has evidence of validity and reliability of the scores.

Keywords. Perfectionism, children, validation, reliability, adaptation.

${ }^{1}$ José Ventura-León.Universidad Privada del Norte, Perú. Dirección Postal: Lima 05, Perú. E-mail: jventuraleon@gmail.com ${ }^{2}$ Stefani Jara-Avalos. Universidad Privada del Norte, Perú. E-mail: stefani.jara.avalos@gmail.com

${ }^{3}$ Carmen Garcia-Pajuelo. Universidad Privada del Norte, Perú. E-mail: carmen_gp_96@hotmail.com

${ }^{4}$ Carlos Ortiz-Saenz. Universidad Privada del Norte, Perú. E-mail: cortizsaenz@gmail.com

\section{(c) (1) (9)}

Esta obra está bajo una licencia de Creative Commons Reconocimiento-NoComercial-SinObraDerivada 4.0 Internacional. 


\section{Introducción}

En los últimos años, algunos problemas psicológicos, reconocidos como propios de la etapa adulta, han comenzado a emerger en la población infantil. Como ejemplo: estrés (Castillo, 2016), depresión (Borges, dos Santos, \& Aparecida, 2016; Collazos \& Jiménez, 2016), ansiedad (González \& Timon, 2016; Orellana \& Chimbo, 2016) y perfeccionismo (Aguilar \& Castellanos, 2016); este último considerado una temática relativamente nueva a investigar (Morris \& Lomax, 2014) en Latinoamérica (Oros, 2003; Aguilar \& Castellanos, 2017).

El incremento en los estudios acerca del perfeccionismo puede estar sustentado por ser un factor de riesgo para trastornos mentales como ideaciones suicidas, depresión y ansiedad (Flett et al., 2016). De acuerdo con cifras internacionales, se estima que entre el 25\% y 30\% de personas jóvenes presenten características de un perfeccionismo maladaptativo (Hawkins, Watt, \& Sinclair, 2006) y 3 de cada 10 adolescentes presentan perfeccionismo maladaptativo (Flett et al., 2016).

En ese sentido, el perfeccionismo debe ser explorado desde los primeros años de vida, debido a que, estudios longitudinales predicen su implicancia en el compromiso escolar (Damian, Stoeber, Negru-Subtirica, \& Băban, 2017) e indican que esfuerzos por ser perfeccionista o los altos estándares son predictores de la dimensión de escrupulosidad del "Big Five" (Stoeber, Otto, \& Dalbert, 2009) y convierte a las personas en vulnerables para el desarrollo de un trastorno depresivo (Hewitt, Flett, \& Ediger, 1996). En ese contexto, resulta oportuno validar una escala de perfeccionismo en niños peruanos, por la implicancia en la salud mental y las pocas investigaciones en Latinoamérica (Oros, 2003; Aguilar \& Castellanos, 2017).

El perfeccionismo es un rasgo inadaptado de la personalidad cuando se expresa en altos niveles (Flett, Hewitt, Oliver, \& Macdonald, 2002) e históricamente ha sido abordado de forma negativa (Hollender, 1965; Patch, 1984), describiendo dos grandes elementos: (a) autoimponerse estándares altos o inalcanzables; (b) autoevaluación negativa ante los errores y preocupación por cometerlos (Burns, 1980; Frost, Marten, Lahart, \& Rosenblate, 1990; Shafran, Cooper, \& Fairburn, 2002; Oros \& Vargas, 2016; Slaney, Rice, Mobley, Trippi, \& Ashby , 2001). Ambos elementos producen displacer e insatisfacción en la consecución de los logros alcanzados (Slade \& Owens, 1998). A pesar de ello, existe una visión opuesta, que señala que existen características positivas en las personas y sentido de logro por las tareas completadas (Hamachek, 1978), lo cual está asociado con el bienestar psicológico (Rhéaume et al., 2000; Terry-Short, Owens, Slade, \& Dewey, 1995), la autoestima y satisfacción personal (Scappatura, Arana, Elizathe, \& Rutsztein, 2011). En ese sentido, el perfeccionista positivo tiene altos niveles de esfuerzo y bajos niveles de preocupación (Stoeber \& Otto, 2006).

Ambas visiones han suscitado una clasificación bipolar en la que es posible encontrar perfeccionismo negativo y positivo (Arana et al., 2010); perfeccionismo activo y pasivo (Moore, 2010); perfeccionismo desadaptativo y adaptativo (Scappatura, Bidacovich, Banasco-Falivelli, \& Rutsztein, 2017), perfeccionismo disfuncional y funcional (Rhéaume et al., 2000), perfeccionismo no patológico y patológico (Kempke et al., 2011). 
En la búsqueda de la explicación de porqué surge el perfeccionismo, se plantea que ocurre por la interacción entre el menor y los progenitores (Flett et al., 2002; Greblo \& Bratko, 2014). Se ha demostrado que cuando las madres tienen altas frecuencias de control, directividad, crítica y conductas perfeccionistas, ponen injustificada presión a sus hijos (Hyson, Hirsh-Pasek, Rescorla, Cone, \& Martell-Boinske, 1991). Asimismo, se muestra evidencia de su relación con otras variables como la depresión (Asseraf \& Vaillancourt, 2015; Huggins, Davis, Rooney, \& Robert, 2008; Hewitt et al., 1996), la formación del autoconcepto (Rice, Kubal, \& Preusser, 2004; Shafran et al., 2002), la baja autoestima (Rutsztein, Scappatura, \& Murawski, 2007), los altos niveles ansiedad

Tabla 1

Características sociodemográficas de la muestra total y submuestras

\begin{tabular}{|c|c|c|c|c|c|c|}
\hline \multirow[t]{2}{*}{ Variables } & \multicolumn{2}{|c|}{ Total $(N=1084)$} & \multicolumn{2}{|c|}{$\operatorname{AFE}(N=200)$} & \multicolumn{2}{|c|}{$\operatorname{AFC}(N=884)$} \\
\hline & $f$ & $\%$ & $f$ & $\%$ & $f$ & $\%$ \\
\hline \multicolumn{7}{|l|}{ Sexo } \\
\hline Mujer & 555 & 51.2 & 108 & 54.0 & 448 & 50.7 \\
\hline Varón & 529 & 48.8 & 82 & 46.0 & 436 & 49.3 \\
\hline \multicolumn{7}{|l|}{ Edades } \\
\hline 7 & 4 & 0.4 & 1 & 0.5 & 3 & 0.3 \\
\hline 8 & 188 & 17.3 & 40 & 20.0 & 148 & 16.7 \\
\hline 9 & 313 & 28.9 & 60 & 30.0 & 253 & 28.6 \\
\hline 10 & 232 & 21.4 & 43 & 21.5 & 189 & 21.4 \\
\hline 11 & 261 & 24.1 & 40 & 20.0 & 221 & 25.0 \\
\hline 12 & 86 & 7.9 & 16 & 8.0 & 70 & 7.9 \\
\hline \multicolumn{7}{|c|}{ Instituciones educativas } \\
\hline 1 & 183 & 16.9 & 28 & 14.0 & 155 & 17.5 \\
\hline 2 & 230 & 21.2 & 37 & 18.5 & 193 & 21.8 \\
\hline 3 & 59 & 5.4 & 10 & 5.0 & 49 & 5.5 \\
\hline 4 & 115 & 10.6 & 24 & 12.0 & 91 & 10.3 \\
\hline 5 & 190 & 17.5 & 40 & 20.0 & 150 & 17.0 \\
\hline 6 & 98 & 9.0 & 17 & 8.5 & 81 & 9.2 \\
\hline 7 & 209 & 19.3 & 44 & 22.0 & 165 & 18.7 \\
\hline \multicolumn{7}{|l|}{ Grado escolar } \\
\hline 3 & 311 & 28.7 & 67 & 33.5 & 244 & 27.6 \\
\hline 4 & 312 & 28.8 & 54 & 27.0 & 258 & 29.2 \\
\hline 5 & 165 & 15.2 & 28 & 14.0 & 137 & 15.5 \\
\hline 6 & 296 & 27.3 & 51 & 25.5 & 245 & 27.7 \\
\hline
\end{tabular}


(Essau, Leung, Conradt, Cheng, \& Wong, 2008; Affrunti \& Woodruff-Borden, 2015), la agresividad (García-Fernández, Vicent, Cándido, Gonzálvez, \& Sanmartín, 2016), los afectos negativos (Vicent, Inglés, Gonzálvez, Sanmartín, \& García-Fernández, 2016) y se muestra como un factor de riesgo para adquisición de trastornos alimenticios en la adolescencia (Pamies \& Quiles, 2014).

Pese a ello, son escasos los instrumentos para medir el perfeccionismo infantil. Entre ellos se encuentran cuatro. Primero, el Child-Adolescent Perfectionism Scale (CAPS) (Flett et al., 2000), que mide el perfeccionismo en dos factores: Perfeccionismo socialmente prescrito y Perfeccionismo autorientado (Aguilar \& Castellanos, 2016). Segundo, Adaptive/Maladaptive Perfectionism Scale (AMPS) (Rice \& Preusser, 2002), de origen estadounidense, que presenta cuatro dimensiones: sensibilidad por cometer errores, autoestima contingente, compulsividad y necesidad de admiración (Rice \& Preusser, 2002). Otro instrumento es el Inventario de Perfeccionismo Infantil (IPI) (Lozano, García, Martín, \& Lozano, 2012), que ostenta tres factores: Autovaloración, Autoexigencia y Presión externa. Por último, la Escala de Perfeccionismo Infantil (EPI) (Oros, 2003), de origen argentino, que tiene dos factores: autodemandas y reacciones ante el fracaso. Está compuesta por dieciséis ítems, recientemente validada a la población venezolana, en donde se identificaron cuatro factores (Aguilar \& Castellanos, 2017).

Con base en los supuestos teóricos y la evidencia empírica presentada, la presente investigación tiene como objetivo analizar las propiedades psicométricas de la Escala de Perfeccionismo Infantil (EPI, Oros, 2003) en una muestra de niños peruanos. Se prefirió la EPI por ser, como se dijo, de origen latinoamericano (Aguilar \& Castellanos, 2017) y de esa forma se espera brindar mayor evidencia acerca del perfeccionismo en la región de Sudamérica. En ese sentido, el estudio se justifica a partir de cuatro aspectos fundamentales: primero, el no contar con una escala para medir el perfeccionismo en la población infantil peruana; segundo, la escasa o nula información acerca del perfeccionismo infantil en el Perú; tercero, a nivel teórico, se podrá examinar si la estructura factorial permanece estable o presenta variaciones debido a factores culturales (Fernández, Pérez, Alderete, Richaud, \& Fernández, 2010; Muñiz, Elosua, \& Hambleton, 2013); cuatro, a nivel práctico, la brevedad de la EPI permitirá una rápida forma de identificar el perfeccionismo, en estudios epidemiológicos e investigaciones en relación con otras variables en el Perú.

\section{Método}

Diseño

El presente estudio corresponde a un diseño instrumental, debido a que consiste en el estudio de las propiedades psicométricas de un test autoinformado (Montero \& León, 2007).

\section{Participantes}

Se contó con la participación de 1084 niños: 555 mujeres y 529 varones; pertenecientes a siete instituciones educativas, privadas y públicas; con edades comprendidas entre los 7 y 12 años $(M=9.75 ; D E=1.23)$, obtenidos por muestreo no probabilístico de tipo intencional. Es importante señalar que se dividió la muestra en dos submuestras; una para 
el AFE y otra para el AFC (véase tabla 1). En vista de que se trabajó con niños, se siguieron procedimientos éticos como el consentimiento informado. Este fue administrado por el departamento psicológico de las instituciones educativas en estudio.

\section{Instrumento}

Se utilizó la Escala de Perfeccionismo Infantil (EPI, Oros, 2003) elaborada en argentina (Anexo 1) y está compuesta por dieciséis ítems. Las alternativas de respuesta en los primeros ocho ítems van del 1 al 3 con expresiones de "No lo pienso", "Lo pienso a veces", "Lo pienso". Por otro lado, los ítems del 9 al 16 reciben las expresiones: "No", "A veces", "Sí”. La EPI presenta evidencia de validez mediante Análisis Factorial Exploratorio (AFE). Las medidas de adecuación muestral fueron optimas $(\mathrm{KMO}=.82$, Bartlett $\mathrm{P}<.01)$. Se utilizó el grafico de sedimentación para la determinación del número de factores que indicó la presencia de dos variables latentes.

El Factor 1 se denominó "Autodemandas" compuesto por los ocho primeros ítems, que se refieren a exigencias internas que se realiza el niño acerca de su desempeño escolar; mientras que, el factor 2, "Reacción ante el fracaso", se conforma por los ocho últimos ítems e involucra la forma en que el niño reacciona ante el fracaso, equivocaciones o error. Ambos factores explican el $41.1 \%$ de varianza del modelo. La fiabilidad fue estimada por medio del alfa de Cronbach $(\alpha=.814)$ siendo considerada buena (Cicchetti, 1994).

\section{Procedimiento}

Inicialmente, se realizó una entrevista semi-estructurada con una muestra de diez niños, con la finalidad de comprobar la comprensión de los ítems. Si bien el idioma es español, los temas culturales pueden afectar las interpretaciones (Chahín-Pinzón \& Brinez, 2011); no obstante, no se efectuaron cambios, de esa forma se previno la presencia de algún sesgo en el ítem a causa de temas lingüísticos (Van de Vijver \& Hambleton, 1996). La escala fue aplicada de forma colectiva en siete instituciones educativas en el horario y fecha pactada bajo la modalidad presencial.

Los investigadores se pusieron en contacto con las autoridades de la Institución Educativa para administrar las pruebas como parte de las evaluaciones psicopedagógicas anuales del departamento psicológico. Así, la institución solicitó autorización a los padres de los niños mediante un formato de consentimiento informado. Finalmente, con el objetivo de estandarizar el proceso de aplicación, se leyeron las instrucciones de igual forma en cada una de las instituciones educativas y se contó con dos o tres evaluadores por aula, para aclarar dudas y supervisar el proceso de aplicación.

\section{Estrategia de análisis}

El análisis de datos se efectuó por medio del programa estadístico "R" versión 3.1.2 (R Development Core Team, 2007), software de acceso libre. Además, se efectuó en cuatro etapas que se desarrollarán a continuación. En primer lugar, se analizaron los ítems con base en los estadísticos descriptivos: media aritmética, desviación estándar, asimetría y curtosis (Hair, Anderson, Tatham, \& Black, 2005). En segundo lugar, se realizó el Análisis Factorial Exploratorio (AFE), técnica estadística que reduce datos por la correlación entre sus elementos (Reise, Waller, \& Comrey, 2000). Por esa razón, se verifican las medidas de 
adecuación el Kaiser-Meyer-Olkin (KMO), cuyos valores próximos a la unidad sugieren que los indicadores son explicados por un factor subyacente (Kaiser, 1974) y el test de esfericidad de Barlett cuya significancia estadística rechaza la idea de una matriz de identidad; ambos elementos sugieren que es posible realizar el AFE (Hair, et al., 2009). El método de extracción fue mínimos cuadrados no ponderados (Costello \& Osborne, 2009). El método de rotación fue Promin por la relación entre los factores (Lorenzo-Seva, 1999). La pertenencia de un ítem en un factor es considerada con cargas superiores a .30 (Nunnally, 1987; Kline, 2010). Para la determinación de número de factores se utilizó el Análisis Paralelo (Timmerman \& Lorenzo-Seva, 2011; Enzmann, 1997).

Tabla 2

Análisis preliminar de los items.

\begin{tabular}{lcccc}
\hline \multicolumn{1}{c}{ Items } & $M$ & $\sigma$ & $g^{1}$ & $g^{2}$ \\
\hline 1. Necesito ser el mejor & 2.280 & .756 & -0.517 & -1.081 \\
2. Tengo que ser el mejor alumno & 2.315 & .725 & -0.562 & -0.925 \\
3. Debo ganar siempre & 1.910 & .716 & 0.134 & -1.034 \\
4. No puedo cometer errores & 2.110 & .780 & -0.195 & -1.324 \\
5. Mis trabajos deben ser mejores que los demás & 1.800 & .775 & 0.363 & -1.245 \\
6. No debo perder cuando juego con mis amigos & 1.665 & .770 & 0.657 & -1.018 \\
7. Debo ser el mejor de la clase & 2.105 & .764 & -0.180 & -1.262 \\
8. Debo ser el primero en terminar las tareas escolares & 2.035 & .796 & -0.063 & -1.412 \\
9. Cuando pierdo me siento mal & 1.870 & .750 & 0.218 & -1.192 \\
10. Pienso mucho en las equivocaciones que tuve & 2.345 & .732 & -0.642 & -0.886 \\
11. Me siento muy mal cada vez que pierdo en algún juego & 1.640 & .755 & 0.706 & -0.913 \\
o deporte & & & & \\
12. Me cuesta perdonarme cuando me equivoco & 1.985 & .822 & 0.028 & -1.510 \\
13. Me enojo cuando no logro lo que quiero & 1.745 & .755 & 0.462 & -1.113 \\
14. Me critico mucho a mi mismo & 1.475 & .727 & 1.183 & -0.110 \\
15. Me siento culpable cuando cometo algún error & 2.165 & .823 & -0.315 & -1.449 \\
16. Me insulto cada vez que cometo un error & 1.480 & .714 & 1.148 & -0.119 \\
\hline Nota. Mo & & & \\
\hline
\end{tabular}

Nota. $M=$ Media; $\sigma=$ Desviación Estándar; $g^{1}=$ Asimetría; $g^{2}=$ Curtosis

En tercer lugar, se realizó el Análisis Factorial Confirmatorio (AFC); ese sentido, se comprobó la normalidad multivariada de los datos mediante el coeficiente de Mardia (1970). Del mismo modo, se siguieron las recomendaciones de Mueller y Hancock (2008) acerca del reporte de los índices de bondad de ajuste del modelo: Chi-cuadrada, el ratio de Chi-cuadrado entre los grados de libertad $\left[\chi^{2} / g l\right]$ valores $<2$ indican buen ajuste (Tabachnick \& Fidell, 2007); el Error Cuadrático Medio de Aproximación [RMSEA], un valor $<.06$ indican un buen ajuste (Hu \& Bentler, 1999). La raíz residual estandarizada 
Tabla 3

\begin{tabular}{|c|c|c|c|}
\hline Ítems & F 1 & $\mathrm{~F} 2$ & $b^{2}$ \\
\hline 1 & -.079 & .682 & .437 \\
\hline 2 & -.220 & .779 & .545 \\
\hline 3 & .191 & .662 & .557 \\
\hline $4^{*}$ & .118 & .246 & .093 \\
\hline 5 & .094 & .683 & .516 \\
\hline 6 & .198 & .461 & .311 \\
\hline 7 & -.166 & .836 & .637 \\
\hline 8 & -.003 & .480 & .230 \\
\hline 9 & .388 & -.017 & .147 \\
\hline $10 *$ & .294 & -.025 & .082 \\
\hline 11 & .444 & .190 & .288 \\
\hline $12 *$ & .267 & .208 & .151 \\
\hline 13 & .448 & .024 & .208 \\
\hline 14 & .621 & -.078 & .361 \\
\hline 15 & .443 & -.070 & .181 \\
\hline 16 & .759 & -.083 & .542 \\
\hline \multicolumn{4}{|l|}{ Carga $/ h^{2}$ media } \\
\hline Autovalor & 4.196 & 2.274 & \\
\hline$\%$ de varianza & $40.4 \%$ & & \\
\hline F1 & 1 & & \\
\hline $\mathrm{F} 2$ & .322 & & \\
\hline A & .733 & & \\
\hline$\Omega$ & .761 & & \\
\hline
\end{tabular}

Nota. F1 = Reacción ante el fracaso; F2 = Autodemandas; h $^{2}=$ Comunalidad antes de rotación; $*=$ ítems $<.30$

cuadrática media [SRMR], cuyo valor < .08 indica buen ajuste (Hu \& Bentler, 1999; Browne \& Cudeck, 1992). El índice de ajuste incremental [CFI] $\geq .95$ indica un buen ajuste (Hu \& Bentler, 1999) y el criterio de Akaike [AIC] cuyo modelo con menor valor en este índice indica un buen ajuste (Caballero, 2011).

En cuarto lugar, se estimó la fiabilidad de las puntuaciones a través del coeficiente Omega ( $\omega)$ por ser recomendado cuando se utiliza el modelo factorial (Mcdonald, 1999; Ventura-León \& Caycho, 2017) y en vista de la correlación entre errores, se utilizó un omega corregido que atenúa el impacto de este evento (Raykov, 2001; Komaroff, 1997). 
Tabla 4

Índices de ajuste estadístico de 3 modelos de la Escala de perfeccionismo $(N=884)$

\begin{tabular}{|c|c|c|c|c|c|c|}
\hline & $\begin{array}{c}\chi^{2} \\
(\mathrm{gl})^{*}\end{array}$ & $\chi^{2} / \mathrm{gl}$ & SRMR & CFI & AIC & $\begin{array}{l}\text { RMSEA } \\
\text { [IC 90\%] }\end{array}$ \\
\hline \multirow[t]{2}{*}{ Modelo 1 (original) } & 363.182 & 3.53 & .049 & .893 & 157.18 & .053 \\
\hline & (103) & & & & & {$[.048, .059]$} \\
\hline \multirow[t]{2}{*}{ Modelo 2 (versión AFE) } & 286.975 & 4.48 & .051 & .894 & 158.97 & .063 \\
\hline & (64) & & & & & {$[.055, .070]$} \\
\hline Modelo 3 (errores correlaciones) & 221.328 & 2.21 & .040 & .951 & 21.32 & .037 \\
\hline e2-e7; e9-e11; e14-e16 & $(100)$ & & & & & {$[.030, .044]$} \\
\hline
\end{tabular}

Tabla 5

Saturaciones de los items en los diversos modelos de la Escala de perfeccionismo $(N=884)$

\begin{tabular}{ccccc}
\hline Ítems & Modelo 1 & Modelo 2 & Modelo 3 & e \\
\hline 1 & .582 & .580 & .580 & 0.199 \\
2 & .599 & .601 & .553 & 0.248 \\
3 & .545 & .547 & .557 & 0.306 \\
4 & .364 & - & .369 & \\
5 & .622 & .620 & .634 & \\
6 & .477 & .476 & .495 & \\
7 & .685 & .686 & .647 & \\
8 & .570 & .569 & .574 & \\
9 & .542 & .580 & .507 & \\
10 & .345 & - & .368 & \\
11 & .519 & .564 & .467 & \\
12 & .452 & - & .469 & \\
13 & .526 & .544 & .537 & \\
14 & .523 & .487 & .475 & \\
15 & .425 & .385 & .439 & \\
16 & .455 & .421 & .395 & \\
$\omega$ & .853 & .846 & $.830^{*}$ & \\
\hline
\end{tabular}

Nota. $\omega=$ Coeficiente omega,$*=$ Omega corregido para errores correlacionados; $\mathrm{e}=$ errores correlacionados del modelo 3 


\section{Resultados}

\section{Análisis preliminar de los items}

En la tabla 2, se presenta los estadísticos descriptivos de los ítems: la media aritmética, desviación estándar, asimetría y curtosis. Se observa que el ítem 10 presenta la mayor media $(M=2.345)$ y el ítem 14 la menor $(M=1.475)$. El ítem 15 cuenta con mayor variabilidad y el ítem 16 la menor. La asimetría y curtosis de los ítems son inferiores +/1.5 (Pérez \& Medrano, 2010; Ferrando \& Anguiano-Carrasco, 2010), a excepción del ítem 12 que sobrepasa ligeramente este valor.

\section{Análisis factorial exploratorio}

Inicialmente, se revisaron las medidas de adecuación muestral, tales como: $\mathrm{KMO}=.734$; Bartlett $=620.4, p<.001$. En ese sentido, se procedió a efectuar el AFE. El método de extracción fue el de mínimos cuadrados no ponderados (Costello \& Osborne, 2009). El método de rotación usado fue Promin, por tratarse de una estructura oblicua (LorenzoSeva, 1999). La determinación del número de factores fue mediante el Análisis Paralelo, el cual sugirió que son dos los factores que subyacen a los dieciséis ítems. Los dos factores explican el $40.44 \%$ de la varianza del constructo perfeccionismo. La organización de los ítems se muestra en la tabla 3 , donde se observa que todos los ítems son superiores a .30 (Kline, 2010) a excepción de los ítems: 4, 10, 12. Asimismo, la fiabilidad de la escala es considerada aceptable $(\alpha=.733 ; \omega=.761)$.

\section{Análisis factorial confirmatorio}

Una vez realizado el AFE, se procedió a realizar el AFC (véase tabla 4). Se probaron tres modelos: (a) Modelo 1, dos factores oblicuos con 8 ítems cada uno (versión original); (b) Modelo 2, dos factores oblicuos retirando los ítems 4, 10, 12 por presentar una baja carga factorial en el AFE; (c) Modelo 3, dos factores oblicuos con errores correlacionados basados en el índice de modificación. Cabe mencionar que se calculó el coeficiente de Mardia (1970), el cual indicó un valor de 7.778, este cumplía con el supuesto de normalidad multivariada al ser $<70$ (Rodríguez \& Ruiz, 2008). Así se descartó la atenuación de los datos.

En la tabla 4, además, se presentan los tres modelos antes expuestos. El modelo 1, fue descartado por presentar un índice de ajuste comparativo inferior a .95 (CFI $=.893$ ) (MacCallum \& Austin, 2000). No obstante, el RMSEA y SRMR tuvieron valores aceptables $(<.06$; Hu \& Bentler, 1999). El modelo 2 no mejoró respecto a su índice comparativo $($ CFI $=.894)$ y existió un leve incremento del RMSEA y SRMR, razón por la que se descartó. En ese sentido, el modelo 3 presentó los mejores valores de bondad de ajuste $\left(\chi^{2}(100)=221.328 ; \chi^{2} / \mathrm{gl}=2.21 ; \mathrm{CFI}=.951 ; \mathrm{RMSEA}=.037[.030, .044] ; \mathrm{SRMR}=.040\right)$.

\section{Fiabilidad}

En la tabla 5, se presentan las cargas factoriales y, a partir de ello, se calculan la fiabilidad con el coeficiente $\omega$. Se observa que el modelo 1 presenta la mayor fiabilidad $(\omega=.853$ ), seguido del modelo $2(\omega=.846)$ y finalmente el modelo $3(\omega=.830)$. No obstante, todos los valores pueden ser considerados buenos (Cicchetti, 1994). 


\section{Discusión}

El perfeccionismo puede definirse como los altos estándares de carácter inalcanzable que se impone un individuo y que al no ser cumplidos generan una evaluación negativa ante las equivocaciones cometidas (Burns, 1980; Frost et al., 1990; Shafran et al., 2002; Oros \& Vargas, 2016; Slaney et al., 2001). En ese sentido, el perfeccionismo está altamente implicado con la construcción de un bajo autoconcepto (Rice et al., 2004; Shafran, Cooper, \& Fairburn, 2002) y la adquisición de un trastorno depresivo en la etapa adulta (Hewitt et al., 1996). En este contexto, resulta necesario adaptar y validar una escala de perfeccionismo en la población infantil peruana, que permita detectar y prevenir la ocurrencia de esta problemática.

El presente artículo tuvo como objetivo la validación de la estructura interna y revisión de la fiabilidad de las puntuaciones de la EPI (Oros, 2003) en una población de niños escolarizados de Lima Metropolitana. En ese sentido, los resultados indican que la EPI debe ser interpretada como una escala bidimensional oblicua y que la fiabilidad de las puntuaciones resultan buenas medidas de constructo examinado.

La validación basada en la estructura interna se realizó mediante el modelo factorial. En un primero momento, de modo exploratorio (AFE) que indicó que son dos los factores que subyacen a los dieciséis ítems. Estos hallazgos concuerdan con la versión original (Oros, 2003) y difieren de la versión venezolana (Aguilar \& Castellanos, 2017), que reporta cuatro factores latentes. Esta diferencia es suscitada porque Aguilar y Castellanos (2017) utilizan el método de Componentes Principales que tiene como desventaja la sobrevaloración de las cargas factoriales y no es considerada Análisis Factorial per se (Costello \& Osborne, 2009; Tabachnick \& Fidell, 2007; Ventura-León, 2017a).

En un segundo momento, se utilizó el AFC, modelándose tres estructuras factoriales: Modelo 1, la versión original, dieciséis ítems con dos factores oblicuos; Modelo 2, la versión del AFE, donde se eliminan los ítems 4, 10, 12; Modelo 3, dos factores oblicuos con errores correlacionados. Los resultados indican que el modelo 3, muestra mejores bondades de ajuste.

Así, el coeficiente Chi-cuadrado $\left(\chi^{2}=221.328\right)$ resulta no significativa (Ruiz, Pardo \& San Martín, 2010), con un $\chi^{2} / g l$ próximo a 2 (Tabachnick \& Fidell, 2007), un índice comparativo $\geq .95(\mathrm{CFI}=.951)(\mathrm{Hu} \&$ Bentler, 1999); un índice absoluto $<.08(\mathrm{SRMR}=$ .040), un índice de parsimonia apropiado (RMSEA = .037; AIC = 21.32) (Caballero, 2011; Hu \& Bentler, 1999).

Debido a que el modelo 3, presenta errores correlacionados entre los ítems 2 ("Tengo que ser el mejor alumno") con el ítem 7 ("Debo ser el mejor de la clase"); ítem 9 ("Cuando pierdo me siento mal") con el ítem 11 ("Me siento muy mal cada vez que pierdo en algún juego o deporte"); ítem 14 ("Me critico mucho a mí mismo") con el ítem 16 ("Me insulto cada vez que cometo un error") se puede sugerir que son producto de la similitud en el significado y fraseo de los ítems, de esa forma han provocado una fuente de varianza única (Brown, 2006).

La fiabilidad de la escala fue estimada mediante el coeficiente omega (Mcdonald, 1999; Ventura-León \& Caycho, 2017) y la presencia de errores correlacionados llevó a utilizar 
una corrección para tal condición (Raykov, 2001; Komaroff, 1997) y descartar el uso del Alfa de Cronbach (Huysamen, 2006).

A pesar de la diferencia en el uso de estimadores, los resultados indican que la versión peruana presenta una buena fiabilidad de las puntuaciones $(\omega=.830)$, interpretación que es coherente con el articulo original de Oros (2003) $(\alpha=.814)$ y superior a la versión venezolana $(\alpha=$.757) (Aguilar $\&$ Castellanos, 2017).

Estos resultados tienen implicaciones prácticas debido a que permiten contar con una escala de perfeccionismo para la población infantil, que representa casi la cuarta parte de la población peruana (Instituto Nacional de Estadística e Informática, INEI, 2012), y que, en Lima Metropolitana, una de cada diez personas es infante (INEI, 2014), generando así estudios epidemiológicos o en relación con otras variables.

Sin embargo, la escala no puede ser utilizada fuera del contexto de investigación debido a que, para tales fines, se requiere revisar la especificidad y sensibilidad del instrumento de medida (Cerda \& Cifuentes, 2012). Dentro las implicaciones teóricas, los resultados suponen la necesidad de hacer mayor investigación en Perú acerca del perfeccionismo y validar la EPI en las diversas regiones de este territorio.

A pesar de los favorables hallazgos, el estudio tiene ciertas limitaciones. En primer lugar, la selección no probabilista de la población pueden generar sesgo de muestreo (Lorh, 2000) y se recomienda la utilización de técnicas de muestreo probabilísticas (Pérez-López, 2005). En segundo lugar, el estudio ha concentrado su atención en la validez basada en la estructura interna, por lo que se sugiere la revisión de la EPI desde otras fuentes de validez como la de contenido, relación con otras variables (Ventura-León, 2017b). En tercer lugar, no fue factible estimar la fiabilidad test-retest, por la naturaleza transversal del estudio.

Se concluye que la EPI, como medida breve, ofrece una apropiada evidencia de validez basada en la estructura interna y fiabilidad de las puntuaciones en niños escolarizados de Lima Metropolitana.

\section{Referencias}

Affrunti, N. W., \& Woodruff-Borden, J. (2015). Parental perfectionism and overcontrol: examining mechanisms in the development of child anxiety. Journal of abnormal child psychology, 43(3), 517-529. doi: 10.1007/s10802-014-9914-5

Aguilar, L., \& Castellanos, M. (2017). Validación psicométrica de una escala de perfeccionismo infantil en niños venezolanos. Revista Psicoespacios, 11(18), 3-36. Recuperado de http://revistas.iue.edu.co/index.php/Psicoespacios

Aguilar, L. A., \& Castellanos, M. Y. (2016). Perfeccionismo infantil: una revisión de la literatura. AJAYU, 14(2), 162-226.

Arana, F. G., Scappatura, M. L., Miracco, M., Elizathe, L., Rutsztein, G., \& Keegan, E. (2010). Perfeccionismo positivo/negativo y calidad de vida percibida en estudiantes de psicología, medicina e ingeniería de la ciudad de Buenos Aires. Anuario de investigaciones, 17, 17-24. 
Asseraf, M., \& Vaillancourt, T. (2015). Longitudinal links between perfectionism and depression in children. Journal of abnormal child psychology, 43(5), 895-908. doi: 10.1007/s10802-014-9947-9

Borges, L., Dos Santos, A., \& Aparecida, A. (2016). Sintomatología depresiva y desempeño escolar: un estudio con niños brasileños. Ciencias Psicológicas, 10(2), 189-197.

Brown, T. A. (2006). Confirmatory factor analysis for applied research. New York: Guilford Publications.

Browne, M. W., \& Cudeck, R. (1992). Alternative ways of assessing model fit. Sage focus editions, $21(2), 230-258$.

Burns, D. D. (1980). The perfectionist's script for self-defeat. Psychology Today, 14(6), 34-52.

Caballero, F. (2011). Selección de modelos mediante criterios de información en análisis factorial. Aspectos teóricos y computacionales. (Tesis doctoral). Universidad de Granada. Departamento de Estadística e IO, Granada, España.

Castillo, J. P. (2016). El estrés infantil y sus efectos psicosomáticos en el desempeño escolar. Revista Huella de la Palabra, 2, 48-54.

Cerda, J., \& Cifuentes, L. (2012). Uso de curvas ROC en investigación clínica: Aspectos teórico-prácticos. Revista chilena de infectología, 29(2), 138-141. doi: 10.4067/S071610182012000200003

Chahín-Pinzón, N., \& Briñez, B. (2011). Frecuencia en la práctica de actividad física en la adolescencia y su relación con los niveles de agresividad, impulsividad, y el uso de la Internet y los videojuegos. Psychologia: avances en la disciplina, 5(1), 9- 23. Recuperado de http://www.redalyc.org/articulo.oa?id=297224114002

Cicchetti, D. V. (1994). Guidelines, criteria, and rules of thumb for evaluating normed and standardized assessment instruments in psychology. Psychological assessment, 6(4), 284-290. doi: 10.1037/1040-3590.6.4.284

Collazos, D. \& Jiménez, Á. M. (2016). Depresión infantil: caracterización teórica. Gastrohnup, 15(2), 15-19.

Costello, A. B. \& Osborne, J. W. (2009). Best practices in exploratory factor analysis: Four recommendations for getting the most from your analysis. Pan-Pacific Management Review, 12(2), 131-146.

Damian, L. E., Stoeber, J., Negru-Subtirica, O., \& Băban, A. (2017). Perfectionism and school engagement: A three-wave longitudinal study. Personality and Individual Differences, 105, 179-184.

Enzmann, D. (1997). RanEigen: A program to determine the parallel analysis criterion for the number of principal components. Applied Psychological Measurement, 21(3), 232232. doi: $10.1177 / 01466216970213003$

Essau, C. A., Leung, P. W., Conradt, J., Cheng, H., \& Wong, T. (2008). Anxiety symptoms in Chinese and German adolescents: Their relationship with early learning 
experiences, perfectionism, and learning motivation. Depression and Anxiety, 25(9), 801-810. doi: 10.1002/da.20334

Fernández, A., Pérez, E., Alderete, A., Richaud, M., \& Fernández, M. (2010). ¿Construir o adaptar tests psicológicos? Diferentes respuestas a una cuestión controvertida. Evaluar, 10, $60-74$.

Ferrando, P. J., \& Anguiano-Carrasco, C. (2010). El análisis factorial como técnica de investigación en psicología. Papeles del psicólogo, 31(1), 18-33.

Flett, G. L., Hewitt, P. L., Besser, A., Su, C., Vaillancourt, T., Boucher, D., Munro, Y., Davidson, L. A., \& Gale, O. (2016). The Child-Adolescent Perfectionism Scale: Development, psychometric properties, and associations with stress, distress, and psychiatric symptoms. Journal of Psychoeducational Assessment, 34(7), 634-652. doi: 10.1177/0734282916651381

Flett, G. L., Hewitt, P. L., Oliver, J. M., \& Macdonald, S. (2002). Perfectionism in children and their parents: A developmental analysis. En G. L. Flett \& P. L. Hewitt (Eds.), Perfectionism: Theory, research, and treatment (pp. 89-132). Washington, DC: American Psychological Association.

Flett, G., Hewitt, P., Besser, A., Su, C., Vaillancourt, T., Boucher, D., Munro, Y., Davidson, L., \& Gale, O. (2000). The Child-Adolescent Perfectionism Scale. Journal of Psychoeducational Assessment, 34(7) $634-652$.

Frost, R. O., Marten, P., Lahart, C., \& Rosenblate, R. (1990). The dimensions of perfectionism. Cognitive therapy and research, 14(5), 449-468. doi: 10.1007/ BF01172967

García-Fernández, J., Vicent, M., Cándido J., Gonzálvez, C., \& Sanmartín, R (2016). Relationship between socially prescribed perfectionism and aggressive behaviour during late childhood. European Journal of Education and Psychology 10(1), 15-22. doi: 10.1016/j.ejeps.2016.10.003

González, E. K., \& Timon, K. D. (2016). Relación entre tipos de colegio y niveles de ansiedad en una muestra de escolares colombianos. (Tesis de pregrado). Universitaria Iberoamericana, Bogotá, Colombia.

Greblo, Z., \& Bratko D. (2014). Parents' perfectionism and its relation to child rearing behaviors. Scandinavian Journal of Psychology, 55(2), 180-185. doi: 10.1111/sjop.12116

Hair, J. F., Anderson, R. E., Tatham, R. L., \& Black, W. C. (2005). Análisis multivariante. Madrid: Pearson.

Hamachek, D. E. (1978). Psychodynamics of normal and neurotic perfectionism. Psychology: A Journal of Human Behavior, 15(1), 27-33.

Hawkins, C. C., Watt, H. M. G., \& Sinclair, K. E. (2006). Psychometric properties of the Frost Multidimensional Perfectionism Scale with Australian adolescent girls: Clarification of multidimensionality and perfectionist typology. Educational and Psychological Measurement, 66(6), 1001-1022. doi: 10.1177/0013164405285909 
Hewitt, P. L., Flett, G. L., \& Ediger, E. (1996). Perfectionism and depression: Longitudinal assessment of a specific vulnerability hypothesis. Journal of Abnormal Psychology, 105(2), 276-280. doi: 10.1037/0021-843X.105.2.276

Hollender, M. H. (1965). Perfectionism. Comprehensive Psychiatry, 6(2), 94-103. doi: 10.1016/S0010-440X(65)80016-5

Hu, L. T., \& Bentler, P. M. (1999). Cutoff criteria for fit indexes in covariance structure analysis: Conventional criteria versus new alternatives. Structural equation modeling: a multidisciplinary journal, 6(1), 1-55. doi: 10.1080/10705519909540118

Huggins, L., Davis, M., Rooney, R., \& Robert, K. (2008). Socially Prescribed and Self Oriented Perfectionism as Predictors of Depressive Diagnosis in Preadolescents. Australian Journal of Guidance \& Counselling, 18(2), 182-194. doi: 10.1375/ ajgc.18.2.182

Huysamen, G. K. (2006). Coefficient alpha: unnecessarily ambiguous; unduly ubiquitous. SA Journal of Industrial Psychology, 32(4), 34-40.

Hyson, M. C., Hirsh-Pasek, K., Rescorla, L., Cone, J., \& Martell-Boinske, L. (1991). Ingredients of parental "pressure" in early childhood. Journal of Applied Developmental Psychology, 12(3), 347-365. doi: 10.1016/0193-3973(91)90005-O

Instituto Nacional de Estadística e Informática, INIE. (2012). Nota de Prensa Nº62: En El Perú 23 de cada 100 Habitantes son Niños. Recuperado de http:/ /andina.pe/agencia/ noticia.aspx?id=407049

Instituto Nacional de Estadística e Informática, INIE. (2014). Una mirada a Lima Metropolitana. Recuperado de https://www.inei.gob.pe/media/MenuRecursivo/ publicaciones_digitales/Est/Lib1168/libro.pdf

Kaiser, H. F. (1974). An index of factorial simplicity. Psychometrika, 39(1), 31-36. doi: 10.1007/BF02291575

Kempke, S., Van Houdenhove, B., Luyten, P., Goossens, L., Bekaert, P., \& Van Wambeke, P. (2011). Unraveling the role of perfectionism in chronic fatigue syndrome: is there a distinction between adaptive and maladaptive perfectionism? Psychiatry research, 186(2), 373-377. doi: 10.1016/j.psychres.2010.09.016

Kline, R. B. (3rd Ed.). (2010). Principles and practice of structural equation modeling. New York: Guilford Press.

Komaroff, E. (1997). Effect of simultaneous violations of essential tau-equivalence and correlated errors on coefficient alpha. Applied Psychological Measurement, 21(4), 337-348. doi: 10.1177/01466216970214004

Lorenzo-Seva, U. (1999). Promin: A method for oblique factor rotation. Multivariate Behavioral Research, 34(3), 347-365. doi: 10.1207/S15327906MBR3403_3

Lorh, S. (2000). Muestreo: Diseño y análisis. Ciudad de México, México: Internacional Thomson Editores. 
Lozano, L., García, E., Martín, M., \& Lozano, L. (2012). Desarrollo y validación del Inventario de Perfeccionismo Infantil (I.P.I.). Psicothema, 24(1), 149-155.

MacCallum, R. C., \& Austin, J. T. (2000). Applications of structural equation modeling in psychological research. Annual review of psychology, 51(1), 201-226. doi: 10.1146/ annurev.psych.51.1.201

Mardia, K. V. (1970). Measures of multivariate skewness and kurtosis with applications. Biometrika, 57(3), 519-530. doi: 10.1093/biomet/57.3.519

McDonald, R. P. (1999). Test theory: A unified approach. Mahwah, NJ: Lawrence Erlbaum.

Montero, I., \& León, O. G. (2007). A guide for naming research studies in Psychology. International Journal of clinical and Health psychology, 7(3), 847-862.

Moore, K. A. (2010). Gender and the differential effects of active and passive perfectionism on mathematics anxiety and writing anxiety. Cognition, Brain, \& Behavior: An Interdisciplinary Journal, 14(4), 333-345.

Morris, L., \& Lomax, C. (2014). Assessment, development, and treatment of childhood perfectionism: A systematic review. Child and Adolescent Mental Health, 19(4), 225234. doi: $10.1111 /$ camh.12067

Mueller, R. O., \& Hancock, G. R. (2008). Best practices in structural equation modeling. In J. W. Osborne (Ed.), Best practices in quantitative methods. Thousand Oaks, CA: Sage Publications, Inc.

Muñiz, J., Elosua, P., \& Hambleton, R. K. (2013). Directrices para la traducción y adaptación de los tests: segunda edición. Psicothema, 25(2), 151-157 doi: 10.7334/ psicothema2013.24

Nunnally, J. C. (1987). Teoría psicométrica. Ciudad de México, México: Editorial Trillas.

Orellana, D, \& Chimbo, F. (2016). La ansiedad infantil en los niños de primer año de educación general básica. (Tesis de pregrado). Universidad de Cuenca, Ecuador.

Oros, L., \& Vargas, J. (2016). Perfeccionismo infantil: normalización de una escala argentina para su evaluación. Acción Psicológica, 13(2), 117-126. doi: 10.5944/ap.13.2.17822

Oros, L. (2003). Medición del perfeccionismo infantil: desarrollo y validación de una escala para niños de 8 a 13 años de edad. Revista Iberoamericana de Diagnóstico y Evaluación Psicológica, 16(2), 99-112.

Pamies, L., \& Quiles, Y. (2014). Perfeccionismo y factores de riesgo para el desarrollo de trastornos alimentarios en adolescentes españoles de ambos géneros. Anales de Psicología, 30(2), 620-626. doi: 10.6018/analesps.30.2.158441

Patch, A. R. (1984). Reflections on perfection. American Psychologist, 39(4), 386-390. doi: 10.1037/0003-066X.39.4.386

Pérez, E. R., \& Medrano, L. (2010). Análisis factorial exploratorio: bases conceptuales y metodológicas. Revista Argentina de Ciencias del Comportamiento, 2(1), 58-66. 
Pérez-López, C. (2005). Muestreo estadístico: conceptos y problemas resueltos. Madrid, España: Pearson Prentice Hall.

R Development Core Team. (2007). R: A language and environment for statistical computing. Viena, Austria: R Foundation for Statistical Computing [programa informático] [en línea] Recuperado de: http://cran.r-project.org/

Raykov, T. (2001). Bias of coefficient afor fixed congeneric measures with correlated errors. Applied psychological measurement, 25(1), 69-76. doi: 10.1177/01466216010251005

Reise, S. P., Waller, N. G., \& Comrey, A. L. (2000). Factor analysis and scale revision. Psychological Assessment, 12(3), 287-297.

Rhéaume, J., Freeston, M. H., Ladouceur, R., Bouchard, C., Gallant, L., Talbot, F., \& Vallieres, A. (2000). Functional and dysfunctional perfectionists: Are they different on compulsive-like behaviors? Behavior Research and Therapy, 38(2), 119-128.

Rice, K. G., \& Preusser, K. J. (2002). The adaptive/maladaptive perfectionism scale. Measurement and Evaluation in Counseling and Development, 34(4), 210-222.

Rice, K. G., Kubal, A. E., \& Preusser, K. J. (2004). Perfectionism and children's selfconcept: Further validation of the Adaptive/Maladaptive Perfectionism Scale. Psychology in the Schools, 41(3), 279-290. doi: 10.1002/pits.10160

Rodríguez, M., \& Ruiz, M. (2008). Atenuación de la asimetría y de la curtosis de las puntuaciones observadas mediante transformaciones de variables: incidencia sobre la estructura factorial. Psicológica, 29(2), 205-227.

Ruiz, M. A., Pardo, A., \& San Martín, R. (2010). Modelos de ecuaciones estructurales. Papeles del psicólogo, 31(1), 34-45.

Rutsztein, G., Scappatura, L., \& Murawski, B. (2007). Perfeccionismo y baja autoestima a través del continuo de los trastornos alimentarios en adolescentes mujeres de Buenos Aires. Revista Mexicana de Trastornos Alimentarios, 5(1), 39-49. doi: 10.1590/1516-4446-2015-1723

Scappatura, M. L., Arana, F., Elizathe, L., \& Rutsztein, G. (2011). Perfeccionismo adaptativo y desadaptativo en trastornos alimentarios: un estudio de revisión. Anuario de investigaciones, 18, 81-88.

Scappatura, M. L., Bidacovich, G., Banasco-Falivelli, M. B., \& Rutsztein, G. (2017). Dimensiones del perfeccionismo (adaptativo y desadaptativo) en adolescentes con trastorno de la conducta alimentaria. Revista Mexicana de Trastornos Alimentarios, 8(2), 131-141.

Shafran, R., Cooper, Z., \& Fairburn, C. G. (2002). Clinical perfectionism: A cognitivebehavioural analysis. Behaviour research and therapy, 40(7), 773-791. doi: 10.1016/ S0005-7967(01)00059-6

Slade, P. D., \& Owens, R. G. (1998). A dual process model of perfectionism based on reinforcement theory. Behavior modification, 22(3), 372-391. doi: $10.1177 / 01454455980223010$ 
Slaney, R. B., Rice, K. G., Mobley, M., Trippi, J., \& Ashby, J. S. (2001). The revised almost perfect scale. Measurement and evaluation in counseling and development, 34(3), 130-145.

Stoeber, J., \& Otto, K. (2006). Positive conceptions of perfectionism: Approaches, evidence, challenges. Personality and social psychology review, 10(4), 295-319. doi: 10.1207/s15327957pspr1004_2

Stoeber, J., Otto, K., \&Dalbert, C. (2009). Perfectionism and the Big Five: Conscientiousness predicts longitudinal increases in self-oriented perfectionism. Personality and Individual Differences, 47(4), 363-368. doi: 10.1016/j.paid.2009.04.004

Tabachnick, B. G., \& Fidell, L. S. (5th ed.). (2007). Using multivariate statistics. New York: HarperCollins College Publisher.

Terry-Short, L., Owens, R., Slade, P., \& Dewey, M. (1995). Positive and negative perfectionism. Personality and Individual Differences, 18(5), 663-668. doi: 10.1016/0191-8869(94)00192-U

Timmerman, M. E., \& Lorenzo-Seva, U. (2011). Dimensionality assessment of ordered polytomous items with parallel analysis. Psychological methods, 16(2), 209-220. doi: $10.1037 / \mathrm{a} 0023353$

Van de Vijver. F. J. R., \& Hambleton, R. K. (1996). Translating tests: Some practical guidelines. European Psychologist, 1, 89-99. doi: 10.1027/1016-9040.1.2.89

Ventura-León, J. L. (2017a). ¿Componentes principales o factores comunes?: Comentarios al artículo de Hederich-Martínez y Caballero-Domínguez (2016). CES Psicología, 10(1), 146-147. doi: 10.21615/cesp.10.1.9

Ventura-León, J. L. (2017b). ¿Existen los instrumentos validos? Un debate necesario. Gaceta Sanitaria, 31(1), 71-71. doi: 10.1016/j.gaceta.2016.09.007

Ventura-León, J. L., \& Caycho, T. (2017). El coeficiente Omega: un método alternativo para la estimación de la confiabilidad. Revista Latinoamericana de Ciencias Sociales, Niñery Juventud, 15(1), 625-627.

Vicent, M., Inglés, C. J., Gonzálvez, C., Sanmartin, R., \& García-Fernández, J. M. (2016). Perfeccionismo socialmente prescrito y afecto en la infancia. International Journal of Developmental and Educational Psychology. Revista INFAD de Psicología, 1(1), 333-340. doi: 10.17060/ijodaep.2016.n1.v1.183

Recibido: 29 de agosto, 2017

Aceptado: 31 de enero, 2018 


\section{Anexo 1}

EPI

Las frases de este cuestionario muestran algunos pensamientos que pueden tener los chicos de tu edad.

- Si muchas veces se te ocurre lo que dice la frase, pon una cruz donde dice "lo pienso"

- Si lo piensa a veces, pon una cruz donde dice "lo pienso a veces"

- Si nunca piensa lo que dice la frase, pon una cruz donde dice "no lo pienso"

\begin{tabular}{|l|c|c|c|}
\hline & $\begin{array}{c}\text { LO } \\
\text { PIENSO }\end{array}$ & $\begin{array}{c}\text { LO } \\
\text { PIENSO } \\
\text { A VECES }\end{array}$ & $\begin{array}{c}\text { NO LO } \\
\text { PIENSO }\end{array}$ \\
\hline 1. Necesito ser el mejor & 3 & 2 & 1 \\
\hline 2. Tengo que ser el mejor alumno & 3 & 2 & 1 \\
\hline 3. Debo ganar siempre & 3 & 2 & 1 \\
\hline 4. No puedo cometer errores & 3 & 2 & 1 \\
\hline 5. Mis trabajos deben ser mejores que los demás & 3 & 2 & 1 \\
\hline 6. No debo perder cuando juego con mis amigos & 3 & 2 & 1 \\
\hline 7. Debo ser el mejor de la clase & 3 & 2 & 1 \\
\hline $\begin{array}{l}\text { 8. Debo ser el primero en terminar las tareas } \\
\text { escolares }\end{array}$ & 3 & 2 & 1 \\
\hline
\end{tabular}

A continuación, encontrarás algunas reacciones que suelen tener los niños de tu edad cuando las cosas les salen mal.

- Si te pasa siempre lo que dice la frase, pon una cruz donde dice "sí"

- Si te pasa a veces, pon una cruz donde dice "la veces"

- Si nunca te pasa lo que dice la frase, pon una cruz donde dice "no"

\begin{tabular}{|l|c|c|c|}
\hline & SI & A VECES & NO \\
\hline 9. Cuando pierdo me siento mal & 3 & 2 & 1 \\
\hline 10. Pienso mucho en las equivocaciones que tuve & 3 & 2 & 1 \\
\hline $\begin{array}{l}\text { 11. Me siento muy mal cada vez que pierdo en algún } \\
\text { juego o deporte }\end{array}$ & 3 & 2 & 1 \\
\hline 12. Me cuesta perdonarme cuando me equivoco & 3 & 2 & 1 \\
\hline 13. Me enojo cuando no logro lo que quiero & 3 & 2 & 1 \\
\hline 14. Me critico mucho a mi mismo & 3 & 2 & 1 \\
\hline 15. Me siento culpable cuando cometo algún error & 3 & 2 & 1 \\
\hline 16. Me insulto cada vez que cometo un error & 3 & 2 & 1 \\
\hline
\end{tabular}

